UDC 543.429.23

DOI: $10.17223 / 24135542 / 1 / 9$

\author{
O.A. Mayboroda ${ }^{1}$, E. Nevedomskaya ${ }^{2}$ \\ ${ }^{1}$ Tomsk State University (Tomsk, Russian Federation) \\ ${ }^{2}$ The Netherlands Cancer Institute (Amsterdam, The Netherlands)
}

\title{
On nuclear magnetic resonance, metabolomics and "metabolic individuality"
}

\begin{abstract}
Metabolomics is an attractive methodology for clinical research as it is the most accurate reflection of the actual physiological and biochemical state of the organism. The dynamic and highly "individualized" nature of the metabolome is a strong indication that it could provide the means to make personalized medicine go all the way from an "elusive dream", , via "proof-of-principle", to real application.
\end{abstract}

Key words: NMR; metabolomics; personalized medicine.

In December 1945 an extraordinary event has occurred in the Radiation Laboratory of MIT (Cambridge, Massachusetts). Edward Purcell, Henry Torrey and Robert Pound have detected weak radiofrequency signal generated by the nuclei of atoms in ordinary material, namely $1 \mathrm{~kg}$ of paraffin wax. Almost at the same time, Felix Bloch reported a different experiment in which radio signals from the atomic nuclei in water were observed. These two experiments are commonly considered as the birth of the Nuclear Magnetic Resonance (NMR) [1]. Today, NMR is powerful analytical technology that has found a variety of applications in chemistry, but one could wonder whether any of the "founding fathers" of the method could have foreseen a role NMR took in the modern biology and medicine. Here, we take no notice of the most known "incarnation" of NMR - MRI imaging but concentrate on the role this technique played in development the concept of "metabolic individuality". The successes story of NMR is closely related to the field of metabolomics, a post-genomic discipline aiming to the study of metabolites: the end points and the intermediate products of the metabolism. Metabolomics has its roots in the technological revolutions of the sixties, the impressive breakthrough of the computer industry of the seventies and the re-birth of the holistic views in biology triggered by such major undertakings such as the human genome project. Historically, the understanding of intracellular metabolic pathways and fluxes in model (mostly unicellular) organisms has been a dominant trend in the field. However, the clinical metabolomics, which has emerged as a spin-off of the "mainstream", focuses of the research on the metabolic regulation of the entire organism and aiming to the development of novel diagnostic / prognostic tools, prediction of the patients responses to the treatment, exploration human metabolic individuality et cetera. The "experimental" and clinical metabolomics use similar analytical strategies but they are cordially different in their approaches to experimental design and data treat- 
ment/interpretation. NMR was a key element in the emerging clinical metabolomics as an independent discipline.

The idea of the connection of metabolism and human individuality is not new. It was first proposed and documented in the classical work of Sir Archibald E. Garrod "The incidence of alkaptonuria: a study in chemical individuality", the title of which speaks for itself. "...No two individuals of a species are absolutely identical in bodily structure," Garrod wrote as long ago as in 1902, "neither are their chemical processes carried out on exactly the same lines" [2]. The next progress in the field was made almost 50 years later by Roger J. Williams who demonstrated "evidence indicating that each individual possesses what may be called a "metabolic personality" - that is, a distinctive pattern of metabolic traits" and that these traits are maintained over a period of several months [3].

As many other fundamental ideas, the idea of "metabolic personality" or "metabolic individuality", although not enough appreciated at the time it appeared, came back in the $21^{\text {st }}$ century. In the publications of Assfalg et al. and Bernini et al. the two collaborating groups elaborated and experimentally supported exactly the same basic thoughts - that "metabolic phenotypes" (the name changed slightly 60 years after R.J. Williams) do indeed exist and that they are stable over time $[4,5]$. As the analytical technologies have advanced enormously in the last decades, the analytical basis of the latest research is different from that of R.J. Williams, who had to use a combination of the test available in the contemporary clinical chemistry laboratory. The use of NMR makes it possible to measure a large number of molecules in one run and to obtain precise quantitative information on these molecules. However, as shown by Assfalg et al., the full variety of metabolites assessed by NMR is not necessary to define the individual metabolic patterns - equally good results can be obtained using only a limited set of twelve compounds. The latter are even to a certain extent overlapping with those measured by Williams. The importance of metabolic phenotypes in relation to disease, nutrition and response to various stimuli has been outlined in both studies. All the environment and nutrition influences in, for instance, urine are superimposed on the invariant profiles represented by metabolic phenotypes. The existence and stability of such distinct profiles are related to homeostasis. Homeostasis is defined as "a state of dynamic balance with the variables fluctuating between tolerance limits". The moment when "tolerance limit" is crossed and homeostasis lost can be considered as the moment of the onset of disease [6]. Detecting this occasion might enable early diagnosis, prognosis and possibilities for more successful intervention.

There is an increasing awareness that metabolomics is of great importance for the medicine of the future due to its "personalized" and dynamic nature. However, to make this possible, the present strategies in metabolomics experiment design and data treatment should be changed. Currently metabolomics literature is dominated by "case-control" studies, which both average the effects between individuals and neglect the beneficial dynamic essence of metabolic profiles. Clinical metabolomics is not an exception, though the concept of "dynamic disease" has been around for a considerable time [7]. The advantages and 
the gain in information recovery obtained by dynamic profiling are starting to be recognized, but are not universally applied. In order to generalize its use it is important to change study design, sample collection and data analysis strategies. Metabolomics data generated by NMR and is multivariate by its nature due to the large number of molecular species measured in one run. With the addition of the time dimension the data becomes also multilevel as different levels of variation, for instance, between- and within-individual, can be assessed. A collection of powerful methods for dealing with such data and for separating the levels of variability is thus called multilevel [8-10]. Exploring the between-individual variation allows neglecting the intra-individual changes, which may be nonsystematic day-to-day differences that do not relate to the question of interest. On the other hand, the within-individual block comprises the time-related information and using this is a more personalized approach for data analysis as each person acts as its own control. Taking into account the extremely personalized nature of metabolic profiles and the abundance of very subtle, "silent" perturbations of metabolism, that do not bring the organism out of homeostasis and are not easily noticed, it is a question if healthy profile can be generalized and found for a whole population, or it is a subjective entity, that can be referred only to one individual. If the latter case is true, than the disease is also relative to the individual himself and typical "case-control" study design is of little help. The design of the study aiming to understanding whether "health" is a population or an individual characteristic should contain on the one hand a large number of subjects in order to eliminate the individual-specific variation, and on the other follow them in time to investigate the borders of normality for each person. This, in turn, brings us back to a question whether there is technology ready for the large, epidemiological scale studies. The answer appears to be a simple one: despite an explosive development of mass spectrometry only NMR provides the data of such degree of consistency which enables the acquisition stability over long period of time and cross-laboratory comparison.

To sum up, metabolomics is an attractive methodology for clinical research as it is the most accurate reflection of the actual physiological and biochemical state of the organism. The dynamic and highly "individualized" nature of the metabolome is a strong indication that it could provide the means to make personalized medicine go all the way from an "elusive dream" [11], via "proof-ofprinciple", to real application.

\section{References}

1. Malcolm H. Levitt. Spin Dynamics: Basics of Nuclear Magnetic Resonance. Wiley Press, 2007.

2. Garrod A.E. // The Lancet. 1902. No. 160. P. 1616-1620.

3. Williams R.J., Berry L.J., Beerstecher E. // Proceedings of the National Academy of Sciences of the United States of America. 1949. No. 35. P. 265-271.

4. Assfalg M., Bertini I., Colangiuli D., Luchinat C., Schafer H., Schutz B., Spraul M. // Proceedings of the National Academy of Sciences of the United States of America. 2008. No. 105. P. 1420-1424. 
5. Bernini P., Bertini I., Luchinat C., Nepi S., Saccenti E., Schafer H., Schutz B., Spraul M., Tenori L. // J. Proteome Res. 2009.

6. van der Greef J., Smilde A. // J. of Chemometrics. 2005. No. 19. P. 376-386.

7. Glass L., Mackey M.C. From clocks to chaos : the rhythms of life. Princeton, NJ : Princeton University Press, 1988.

8. Jansen J.J., Hoefsloot H.C.J., van der Greef J., Timmerman M.E., Smilde A.K. // Analytica Chimica Acta. 2005. No. 530. P. 173-183.

9. Westerhuis J.A., van Velzen E.J.J., Hoefsloot H.C.J., Smilde A.K. // Metabolomics. 2010. No. 6. P. 119-128.

10. Nevedomskaya E., Mayboroda O.A., Deelder A.M. // Mol. BioSyst. 2011. No. 7. P. 3214 3222.

11. Lesko L.J. // Clinical Pharmacology \& Therapeutics. 2007. No. 81. P. 807-816.

Mayboroda Oleg A., Center for Proteomics and Metabolomics, Leiden University Medical Center (Leiden, The Netherlands); Department of Chemistry, Tomsk State University (Tomsk, Russian Federation). E-mail: O.A.Mayboroda@lumc.nl

Nevedomskaya Ekaterina, Department of Molecular Pathology, The Netherlands Cancer Institute (Amsterdam, The Netherlands). E-mail: O.A.Mayboroda@lumc.nl

DOI: $10.17223 / 24135542 / 1 / 9$ 